\title{
Personalized Recommendation for Online Retail Applications Based on Ontology Evolution
}

\author{
Rana Alaa \\ Business information system \\ Arab Academy for science, \\ Technology and Maritime \\ Transport \\ Cairo, Egypt \\ ranaalaa.aast@hotmail.com
}

\author{
Mariam Gawich \\ Centre de Recherche Informatique \\ Université Française en Egypte \\ (UFE) \\ Cairo, Egypt \\ mariam.gawish@ufe.edu.eg
}

\author{
Manuel Fernández-Veiga \\ AtlanTTIC Research Center \\ University of Vigo \\ Vigo, Spain \\ mveiga@det.uvigo.es
}

\begin{abstract}
The upcoming generation of World Wide Web is signified in semantic web technology that allows future applications to grasp and connect with numerous knowledge bases. Due to its exclusive function in modeling specific domain, Ontology has been playing an essential role in semantic web development. Recommender systems are an indispensable part of online site, which makes their use of high value in recommending items to users according to their interests. The semantic recommender systems recently aim to accomplish the website ontologies to generate semantic recommendations for users' profiles. Therefore, ontology-based semantic recommender systems are used to develop web recommendation. In this paper a recommendation system architecture based on ontology is proposed to give semantic recommendations for each user profile. The proposed system architecture applies two recommendation techniques, contentbased filtering and collaborative filtering.
\end{abstract}

\section{CCS Concepts}

Information systems $\rightarrow$ Information retrieval $\rightarrow$ Users and interactive retrieval $\rightarrow$ Personalization

\section{Keywords}

Semantic recommender system; reasoning; ontology; intelligent personalization; ontology evolution.

\section{INTRODUCTION}

The number of online customers has increased recently due to the spread of internet usage, e-Commerce and online stores [1]. However, customers still face obstacles while selecting products because of the wide range of variety for viewed products [2]. Recommender system (RS), which are active information filtering systems that give recommendations of products to the customers based relatively on their background preferences and interests, have become very helpful and essentially used in web-based applications such as e-Commerce and e-Services [3, 4]. However, the traditional RS techniques such as collaborative filtering and Permission to make digital or hard copies of all or part of this work for personal or classroom use is granted without fee provided that copies are not made or distributed for profit or commercial advantage and that copies bear this notice and the full citation on the first page. To copy otherwise, or republish, to post on servers or to redistribute to lists, requires prior specific permission and/or a fee. Request permissions from Permissions@acm.org.

ICCTA 2020, April 14-16, 2020, Antalya, Turkey

(C) 2020 Association for Computing Machinery.

ACM ISBN 978-1-4503-7749-2/20/04 ..\$15.00

https://doi.org/10.1145/3397125.3397134 content based suffer from some issues such as, the cold start, the sparsity and the scalability problem. The first problem that the current recommender systems face, especially the collaborative filtering, is the cold start problem. The cold start problem occurs when a new user or new item comes into the system. In this case it is very difficult to provide recommendations for new user because there is not enough information about the user that is available and also for the new items no ratings are usually available. The second problem is the sparsity that occurs when the user does not rate most of the items and the available ratings are sparse. Collaborative filtering suffers from the previous problem because it is depending on the rating matrix in most cases. The third problem that will be tackled in this paper is the scalability problem, which occurs when number of users and items grow. Therefore, the system needs more information in order to give the most accurate recommendations to the users [5]. The fourth problem that faces the current RSs is that the relation between users and objects is not presented in meaningful way.

Therefore, this paper introduces the use of ontology to solve the previous problems. The traditional RSs techniques are concentrated to discover meaningful patterns that may bring out some useful information about customer purchase history. However, the existence of huge amount of historical data stored sometimes the patterns of purchases may not pertain to a specific customer but to a set of customers. The RSs techniques are done when the customers are offline. That's why, the personalization of the website may not be accurate to fit the customer's immediate need, and sometimes the information may already be outdated [6], and by the use of semantic personalized recommender systems based on ontology will discover user's preferences and interests by semantically analyzing their online purchase behaviors in order to provide accurate online recommendations.

This paper presents a recommendation system architecture based on ontology evolution and applies two recommendation techniques; content-based filtering and collaborative filtering.

The rest of the paper is organized as follows; section two discusses the ontology and ontology evolution, section three discusses the ontology-based recommendation system, section four illustrates the ontology reasoning, section five present online retail recommendation system, section six gives summary about the previous work, Section seven presents the proposed recommendation system architecture based on ontology evolution ,section eight shows comparison between recommender systems and finally the conclusion in section nine. 


\section{ONTOLOGY AND ONTOLOGY EVOLUTION}

Ontology is represented in a set of concepts and categories in a subject matter or domain that shows their properties and the relationships between them. What's new about ontology is that it is created automatically from large datasets. It is used to capture and analyze specific knowledge within a domain of interest. Nowadays, ontologies emerged for exploring new approaches towards diverse applications. In this regard, ontologies are deemed to play indispensable role for semantic web. As such, ontologies are manipulated in the area of knowledge management, model integration and information[7].

Ontologies provide wide range of reasoning capabilities, exploitation of queries and explicit imagining of schema. However, information based on ontologies cannot be guaranteed to be stable, same as any other knowledge structure, where they must be renovated and updated from time to time, in order to obtain an adequate answer for queries. As such, ontology development is a dynamic process, starting from its initial step to end of its update, whereby it is noticed that during the use of ontologies, the underlying knowledge behind the domain may change and evolve. Therefore, in order to realize optimal use of ontologies, they have to be updated on continued basis to keep abreast with the spontaneous and frequent changes taking place. The evolution process deals with the ontology growth. In its essence, ontology evolution means upgrading, modifying or changing the ontology whenever it is required to make such change or whenever a change exists in the domain information[7].

Stojanovic and colleagues [8] define Ontology Evolution-as follows:

Ontology evolution is the process of opportune adaptation with the changes in the business requirements. Such evolution is also manifested in the trends in ontology instances and patterns of usage of the ontology-based application along with the steady management and proliferation of these changes to dependent elements [8].

At the present times, ontology evolution is very essential. The main drive for such evolution is the growing number of ontologies in use and the rising costs accompanying the adaptation with the changing requirements [9].

\section{ONTOLOGY BASED RECOMMENDATION SYSTEMS}

Recommender or recommendation systems suggest predictions of preferences or rating that a user would give to an item. Recently, RS have become widely known and frequently used in various applications including news, books, movies, music, search queries, research article and social tags. The use of heterogeneous information can be integrated by ontology and start guidance of recommendation preferences. For user profiling, personalized search and web browsing, ontology-based, recommendation system uses ontology [10].

Ontologies help in broadening recommender systems to an environment of various classes, which allows methods based on knowledge to be used with classical machine learning algorithms. In addition, they have been used regularly in recommender systems accompanied with machine learning, statistical correlations, user profiling and domain specific heuristics. Commercial recommender systems usually either sustain simple product ontologies that can be later applied via heuristics or have a large community of users who are rating content suitable for collaborative filtering actively [5].

\section{ONTOLOGY REASONING}

Reasoning means inference of new information from the statements affirmed by the ontology engineer. The reasoner recognizes the illegal mistakes made by the developer[11]. A "Reasoner" is simply expressed in a semantic reasoner, a reasoning engine or rules engine. It is software that is able to conjecture logical consequences from a set of certain confirmed facts or axioms. The rules of inference are usually specified by means of descriptive language and they flow into forward and backward chaining. Yet, the strategies of the ontology reasoners rely on the forward and backward chaining. Forward chaining is the reasoner's strategy which starts from the known facts and conclude valid inferences. Meanwhile, backward chaining is the reasoner's strategy that starts from a certain fact or a query, for the purpose of verification or finding all possible solutions. Among the large number of reasoners available, are the popular reasoners that suit protégé-Pellet, RACER, FACT++, HermiT[12].

\section{ONLINE RETAIL RECOMMENDATION SYSTEM}

The development of recommender systems has to be stimulated by the rapid growth of information on the internet and the increasing number of items offered on online retail websites. Such huge volume of products offered online creates obstacles to users in selecting the items they need. That's why recommender systems become a part of everyday life.

The recommender system plays vital role in online retail, as follows [13].

1. Increasing the volume of items of products sold, which is the most eminent objective for commercial RSs. This objective will be realized by giving suitable recommendations tailored with users' needs.

2. Selling more diverse items. This is one of the major functions of RS to help users select items that they can't easily find without recommendations.

3. Enhancing user satisfaction. A well designed RS will improve users experience websites, in that the user will find the recommendations interesting and relevant.

4. Increasing user fidelity. The user's loyalty to the website will be achieved after the recommender systems recognizes the customer as an old customer and treats him as a valuable visitor, by giving him recommendations according to his past behavior over the website, e.g., his ratings for his previous items [13].

As these various points indicate the role of recommender system online. So recommender systems are information processing systems that gather various kinds of data to build recommendations according to customer preferences. The next section introduces a proposed recommendation model based on ontology evolution to give each user profile recommendation according to his behavior over the online retail website.

\section{RELATED WORK}

Ade Romadhony, Said Al Faraby and Bambang Pudjoatmodjo focused on the design of good personal recommendations. In their research, the system generates two types of recommendations: personal and item-based [14].

User-based collaborative filtering is implemented to produce personal recommendations. The research implements user-based 
collaborative filtering $(\mathrm{CF})$ to produce personal information and implements item-based collaborative filtering $(\mathrm{CF})$ on items recommend. The evaluation has been taken by conducting a survey on the users. The users should state whether they like the recommendation or not. The results show that most of the users like the item-based recommendations but the personal recommendation is only preferred by the user, who already does some activities, rate or view an item.

Waltercarre proposed a hybrid recommender system based on knowledge and social networks which is presented in the domain of the cinema graphic in [15]. The proposed system users are free to choose the product to buy which their social network will influence on the recommendations directed to them. The proposed system presents a social based contents recommender system that makes use of the semantic web principles to build a mechanism to help users find content that is relevant with their preferences. The proposed hybrid system put both concepts of knowledge and collaborative filtering that allow suggestion to be made based on 1) the semantic linkage between contents users preferences and 2) also inherit recommendations from the users social network. Their research also introduces the concept of "social aperture", by means of which users can decide the impact of social networks will have on the recommendations.

Huichuan proposed a hybrid personalized recommendation model based on users' behaviors context aware, which combines content based filtering with collaborative filtering methods [16]. The research presents a personalized recommendations system based on content based filtering that could effectively recommend information to users by users' interest that already knew, while recommendation system based on collaborative filtering could learn users new interest by users similar action. The researcher designs an online book system to carry out the research and the analysis of personalized recommendation system, the data tested was downloaded from movie lens. The research randomly selects 500 rating data in the data based which include rating of 514 books rated by thirty users.

Pin-yu and chi-Hsuan Proposed a hybrid recommendation strategy of content based and knowledge based that aims to overcome the problems; New Item Problem, the Rating Sparsity Problem, the Limited Content Analysis Problem, the Overspecialization Problem[17]. They propose ontology based adaptive personalized recommender system to solve the problems mentioned. The ontology based adaptive personalized recommender system is implemented in the movie recommendation. The researches adopt the movie ontology then apply the ontology model in the movie ontology and created instances according to the internet movie database (IMDB) via the protégé-OWL application programming interface (API) from the protégé project. The recommendation module is based on an approach of multi-agent personalization. The proposed strategy of the hybrid recommendation techniques is also portable and easy to be ported to other application areas as long as the domain knowledge is given and the searching space is large enough.

Qian and Xianhu Proposed a hybrid user model in which the recommender system based on this model not only holds the advantage of recommendation accuracy in memory based method but also has the scalability as good as model based method[18]. The user model is constructed based on item combination features and demographics information and it focuses on searching for set neighboring users shared with same interests which helps to improve system scalability. To enhance recommendation accuracy each feature in user model is given a different weight when computing the similarity between users. Genetic algorithm is adaptive to learn the weight value of features. A comparison experiment uses performed on movie lens dataset. Then, the experiment shows that proposed algorithm can get recommendations with higher accuracy compared to methods of traditional collaborative filtering and content based filtering combined with collaborative filtering.

\section{PROPOSED RECOMMENDATION SYSTEM ARCHITECTURE BASED ON ONTOLOGY EVOLUTION}

As figure 1 mentions the proposed recommendation system architecture consists of data collection, ontology evolution, ontology reasoning and recommendation techniques.

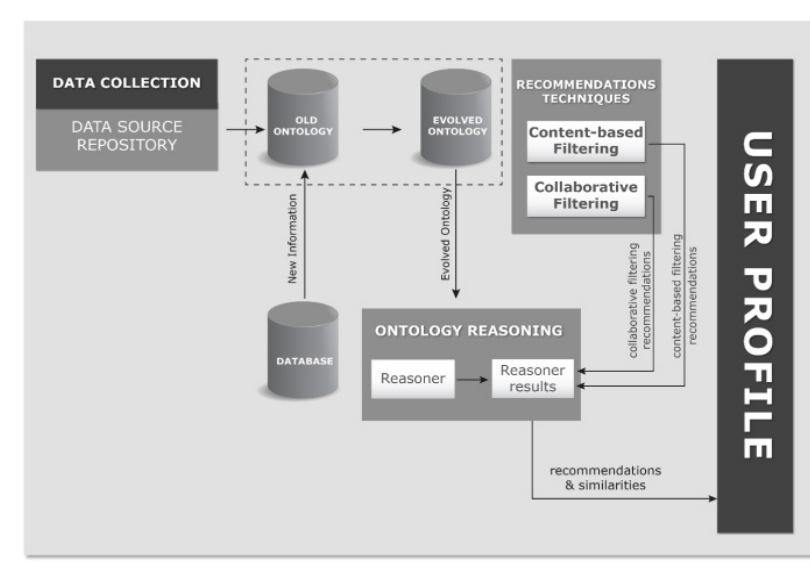

Figure 1 A proposed recommendation system architecture based on ontology evolution

\subsection{Data collection}

In information collector part, the information are collected through data source repository to apply the reasoning techniques to give the suitable recommendations and similarities for the user profiles by the use of the collaborative filtering and content-based filtering recommendation techniques .

The input: the current ontology specification is built by using OWL2 language in protégé with semi-automatic way which is based on the use of celfie plugin that connects the ontology with the dataset that is located in excel file. The celfie will append the ontology with individuals, data properties and object properties defined by the ontology engineer.

\subsection{Ontology evolution}

It is the adaptation of ontology to changes that are appeared in the domain of interest. The adaption should ensure the consistency of ontology and its depending artifacts. These artifacts can be other ontologies, websites and other applications. So the process of adaption of the ontology according to the domain of interest is called ontology evolution[].

The input for ontology evolution is the new source of data from database and the current ontology. Therefore, the output for ontology evolution is the evolved ontology.

\subsection{Reasoner}

The reasoner was applied on three phases: 1) Gathering and processing of the user profiles. 2) Process all defined reasoning 
rules and execute them for the proposed ontology 3) Show the results to user profiles[].

The input for ontology reasoning is the evolved ontology. The output will be the reasoner results that give recommendation and similarities to user profiles according to the collaborative and content-based filtering recommendation techniques.

\subsection{User Profile}

The information about the user, including his/her preferences are usually stored in a personal data structure known as user profile, which can be constructed considering direct feedback from the users, so that the use of profiles to represent the users interests is important for recommendations []ㅡ.

\subsection{Recommendation techniques used}

\subsubsection{Collaborative filtering}

This technique suggests items to users who have similar interests and preferences according to their behaviors in the past [ $[$ ].

\subsubsection{Content based filtering}

This technique relay on item descriptions and generate recommendations from items that are similar to those the target user has liked in the past without directly relying on the preferences of other users[19].

\section{RECOMMENDER SYSTEM COMPARATIVE STUDY}

The following table 1 presents a comparative study between different recommendation architectures with diverse applied recommendation techniques.

Table 1 recommender system comparison [14-17]

\begin{tabular}{|c|c|c|c|c|}
\hline Authors & $\begin{array}{l}\text { Recommendation } \\
\text { techniques }\end{array}$ & $\begin{array}{l}\text { Social } \\
\text { network }\end{array}$ & $\begin{array}{l}\text { Reasoning } \\
\text { techniques }\end{array}$ & $\begin{array}{l}\text { Ontology } \\
\text { evolution }\end{array}$ \\
\hline $\begin{array}{l}\text { Ade } \\
\text { Romadhony } \\
\text { Said } \\
\text { Al Faraby and } \\
\text { Bambang } \\
\text { Pudjoatmodjo }\end{array}$ & $\begin{array}{l}\text { User based (CF) } \\
\text { Item based (CF) }\end{array}$ & No & No & No \\
\hline Waltercarre & $\begin{array}{l}\text { Knowledge } \\
\text { based and }(\mathrm{CF})\end{array}$ & Yes & No & No \\
\hline Huichuan & $\begin{array}{l}\text { Content based } \\
\text { filtering and } \\
\text { collaborative } \\
\text { filtering }\end{array}$ & No & No & No \\
\hline $\begin{array}{l}\text { Pin-yu and chi- } \\
\text { Hsuan }\end{array}$ & $\begin{array}{l}\text { Knowledge } \\
\text { based and content } \\
\text { based filtering }\end{array}$ & No & No & No \\
\hline $\begin{array}{l}\text { Proposed } \\
\text { architecture }\end{array}$ & $\begin{array}{l}\text { Content-based } \\
\text { filtering and } \mathrm{CF}\end{array}$ & No & Yes & Yes \\
\hline
\end{tabular}

Points of comparison that are used to trade off between recommender systems: Recommendation techniques, social network, apply reasoning, applying ontology evolution technique as table (1) shows Ade Romadhony, Said Al Faraby and Bambang Pudjoatmodjo use user based collaborative filtering (CF) and item based collaborative filtering (CF) as recommendation techniques . Waltercarre use Knowledge based and collaborative filtering as recommendation techniques. Waltercarre uses the social network in recommendation. Huichuan use Content based filtering and collaborative filtering as recommendation techniques. Pin-yu and chi-Hsuan use
Knowledge based and content based filtering as recommendation techniques. The proposed architecture propose the use of collaborative filtering and content based filtering as recommendation techniques. The demographic variables are used in making recommendation to find out the similarities between user profile are age and gender .....etc. The proposed architecture applies the use of the reasoning technique to detect the similarities between user profiles and recommend products according to each user profile preferences and past behavior. The proposed architecture use the ontology evolution technique in recommendation to detect the change of behavior of the user over the years and give accurate and updated recommendations to each user.

\section{CONCLUSION}

In this paper a personalized recommendation system architecture based on ontology evolution is proposed to give updated recommendations. It applies two recommendations techniques content based filtering which tries to find similar content among user profiles to know the users preferences to give for each user profile the suitable and the desired recommendations and collaborative filtering that tries to find similar users profiles similar to their interests and actions over the online retail websites to give them the same recommendations according to their behaviors. The proposed recommendation system architecture introduces also the use of the reasoner that generates recommendations according to the user profile description by detecting the similarities between users profiles and give recommendations for each user profile according to his similarity with another user profile and according to his behavior; it recommends products to him that fit his preferences. So the recommendation is done by applying the hybrid recommendation techniques.

In future, the proposed system will take into consideration customer's comments and reviews for the previous recommendation over social media and a social recommendation model based on ontology evolution will be proposed to enhance the recommendation process.

\section{ACKNOWLEDGEMENT}

This work was supported by the European Regional Development Fund (ERDF) and the Galician Regional Government under agreement for funding the atlanTTic Research Center for Telecommunication Technologies (atlanTTic), and by the "Ministerio de Economia, Industria y Competitividad" through the project TEC2017-85587-R of the "Programa Estatal de Investigacin', Desarrollo e Innovación Orientada a los Retos de la Sociedad" (partly financed with FEDER funds).

\section{REFERENCES}

[1] Ying, M. and Hsu, Y. 2014. A commodity search system for online shopping based on ontology and web mining. : International Conference on Software Intelligence Technologies and Applications \& International Conference on Frontiers of Internet of Things 2014 (Hsinchu, Taiwan, 2014), . 6- 11

[2] Widyantoro, D. and Baizal, Z. 2014. A framework of conversational recommender system based on user functional requirements. 2014 2nd International Conference on Information and Communication Technology (ICoICT) (Bandung, Indonesia, 2014), 160-165.

[3] Nguyen, T. et al. 2010. ntology-style web usage model for semantic web applications," in 2010 10th International 
Conference on Intelligent Systems Design and Applications. 2010 10th International Conference on Intelligent Systems Design and Applications (Cairo, Egypt, 2010), 784-789.

[4] Sobhanam, H. and Mariappan, A. 2013. Addressing cold start problem in recommender systems using association rules and clustering technique. 2013 International Conference on Computer Communication and Informatics (Coimbatore, India, 2013), 1-5.

[5] Paiva, F. et al. 2013. A hierarchical architecture for ontology-based recommender systems,. 2013 BRICS Congress on Computational Intelligence and 11th Brazilian Congress on Computational Intelligence (Ipojuca, Brazil, 2013), 362-367.

[6] Primo, T. et al. 2012. User profiles and learning objects as ontology individuals to allow reasoning and interoperability in recommender systems. Proceedings of the 2012 IEEE Global Engineering Education Conference (EDUCON) (Marrakech, Morocco, 2012), 1-9.

[7] T. Sahu. (2015). ONTOLOGY EVOLUTION. Available: https://www.semanticscholar.org/paper/ONTOLOGYEVOLUTION-Seminar-in-Data-and-Knowledge-Sahu

[8] Yildiz.B.2006. "Ontology evolution and versioning," Vienna University of Technology, Karlsplatz, 2006.

[9] Stojanovic.L.2004. "Methods and tools for ontology evolution," 2004.

[10] Dou, D. et al. 2015. Semantic data mining: A survey of ontology-based approaches. Proceedings of the 2015 IEEE 9th International Conference on Semantic Computing (IEEE ICSC 2015) (Anaheim, CA, USA, 2015), 244-251.

[11] Kalaivani.P, et al.2013 "An ontology construction approach for the Domain of poultry science using protégé," arXiv preprint arXiv:1302.5417, 2013.
[12] Abburu.S.2012. "A survey on ontology reasoners and comparison," International Journal of Computer Applications, vol. 57, 2012.

[13] Ricci.F, et al.2011. "Introduction to recommender systems handbook," in Recommender systems handbook, ed: Springer, 2011, pp. 1-35.

[14] Romadhon,A. et al.2013. "Online shopping recommender system using hybrid method," in 2013 International Conference of Information and Communication Technology (ICoICT), 2013, pp. 166-169

[15] Carrer-Neto, $W$. et al.2012. "Social knowledge-based recommender system. Application to the movies domain," Expert Systems with Applications, vol. 39, pp. 10990-11000, 2012.

[16] Liao,H.2013. "A new web service model of hybrid personalized recommendation," in 2013 Ninth International Conference on Natural Computation (ICNC), 2013, pp. 868872.

[17] Pan,P et al.2010. "The development of an ontology-based adaptive personalized recommender system," in 2010 international conference on electronics and information engineering, 2010, pp. V1-76-V1-80.

[18] Wang,Q. et al.2010. "Collaborative filtering recommendation algorithm based on hybrid user model," in 2010 Seventh International Conference on Fuzzy Systems and Knowledge Discovery, 2010, pp. 1985-1990.

[19] Jain,S. et al.2015., "Trends, problems and solutions of recommender system," in International Conference on Computing, Communication \& Automation, 2015, pp. 955-95 